\title{
The Effects of Caffeine on the Long Bones and Testes in Immature and Young Adult Rats
}

\author{
Yoojin Kwak, Hyeonhae Choi and Jaesook Roh \\ Department of Anatomy and Cell Biology, Hanyang University, Seoul, Korea
}

(Received January 31, 2017; Revised March 6, 2017; Accepted March 7, 2017)

\begin{abstract}
This study was to evaluate the age-dependent effects of caffeine exposure on the long bones and reproductive organs using male rats. A total of 15 immature male rats and 15 young adult male rats were allocated randomly to three groups: a control group and two groups fed caffeine with 120 and $180 \mathrm{mg} / \mathrm{kg} / \mathrm{day}$ for 4 weeks. Exposure to caffeine at either dose significantly reduced body weight gain; a proportional reduction in muscle and fat mass in immature animals, whereas a selective reduction in fat mass with relatively preserved muscle mass in young adult animals. The long bones of immature rats exposed to caffeine were significantly shorter and lighter than those of control animals along with decreased bone minerals. However, there was no difference in the length or weight of the long bones in young adult rats exposed to caffeine. Exposure to caffeine reduced the size and absolute weight of the testes significantly in immature animals in comparison to control animals, but not in young adult animals exposed to caffeine. In contrast, the adrenal glands were significantly heavier in caffeine-fed young adult rats in comparison to control animals, but not in caffeine-fed immature rats. Our results clearly show that the negative effects of caffeine on the long bones and testes in rats are different according to the age of the rat at the time of exposure, and might therefore be caused by changes to organ sensitivity and metabolic rate at different developmental stages. Although the long bones and testes are more susceptible to caffeine during puberty, caffeine has negative effects on body fat, bone minerals and the adrenal glands when exposure occurs during young adulthood. There is a need, therefore, to educate the public the potential dangers of caffeine consumption during puberty and young adulthood.
\end{abstract}

Key words: Caffeine, Puberty, Young adulthood, Bone, Testes, Adrenal gland

\section{INTRODUCTION}

Energy drinks have become a popular source of caffeine and a considerable number of adolescents and young adults consume energy drinks regularly (1). Because most energy drinks contain between three- and five-times the amount of caffeine found in other soft drinks, it is easy to exceed the daily recommended dose when consuming energy drinks (2).

Caffeine is a well-known risk factor for osteoporosis and fractures in humans, as it causes bone mineral loss $(3,4)$. Similarly, studies of immature rats have demonstrated that

Correspondence to: Jaesook Roh, Department of Anatomy and Cell Biology, Hanyang University, 222 Wangsimni-ro, Seongdonggu, Seoul 04763, Korea

E-mail: rohjaesook@hanyang.ac.kr

This is an Open-Access article distributed under the terms of the Creative Commons Attribution Non-Commercial License (http:// creativecommons.org/licenses/by-nc/3.0) which permits unrestricted non-commercial use, distribution, and reproduction in any medium, provided the original work is properly cited. chronic exposure to caffeine inhibits both bone mineral mass and long bone growth (5-7). Several clinical and experimental studies suggest that caffeine has negative effects on sex steroids and reproductive organs. For example, prenatal exposure to caffeine inhibits testosterone levels and reduces the weight of sex accessory organs in male rat offspring (8). Likewise, peripubertal exposure to caffeine reduces serum testosterone levels and alters the microarchitecture of the reproductive organs in immature rats (9). Age-related changes to the metabolic rate and body composition, including fat and lean body mass (10), might affect the absorption, distribution and clearance of caffeine. Therefore, children and adolescents might be more vulnerable to caffeine exposure than young adults (11). To the best of our knowledge, however, no studies to date have analyzed whether the effect of exposure to caffeine on long bones and reproductive organs change with age. In addition, clinical data on the effects of caffeine on the reproductive organs in humans are relatively sparse and conflicting (12).

In the current study, we investigated the effects of caf- 
feine on the long bones and testes in immature and young adult male rats by exposing the rats to caffeine every day for four weeks. After four weeks of daily exposure to caffeine, the body mass and composition were recorded. The length, weight, bone mineral content and bone mineral density of the long bones were analyzed, and the testes and sex accessory organs (prostate, seminal vesicles, and epididymis) were weighed, as were the adrenal glands.

\section{MATERIALS AND METHODS}

Animals. Sprague-Dawley rats were obtained from Samtako Biokorea (Osan, Korea). The rats were allowed to acclimatize until 21 (immature) or 64 (young adult) days of age before the experiment started. Each animal was housed in a separate plastic cage under controlled conditions (22 $24^{\circ} \mathrm{C}, 40 \sim 50 \%$ humidity, $12 \mathrm{hr}$ light/dark cycle), with free access to food and water. Animal care was consistent with institutional guidelines, and the Hanyang University (Seoul, Korea) ACUC committee approved all procedures involving animals (HY-IACUC-2013-0110A).

Experimental design. One day before the start of treatment, experimental groups were assigned using the stratified randomization method based upon body weight to eliminate variation in mean body weight between the groups. Caffeine (C0750, Sigma-Aldrich, St. Louis, MO, USA) was dissolved in distilled water $(10 \mathrm{~mL} / \mathrm{kg})$ at concentrations calculated to deliver either $120 \mathrm{mg} / \mathrm{kg}$ body weight per day (group $\mathrm{CF} 1 ; \mathrm{N}=5$ ) or $180 \mathrm{mg} / \mathrm{kg}$ body weight per day (group $\mathrm{CF} 2 ; \mathrm{N}=5$ ). The dosages were based upon previously published literature and range-finding studies to avoid sub-lethal effects at the higher dose. The median lethal dose of caffeine administered orally to rats is $192 \mathrm{mg} / \mathrm{kg}$ (13). The dosages of 120 and $180 \mathrm{mg} / \mathrm{kg}$ per day used in this study induce delayed ossification in rat fetuses (14) and are within the range shown to have no undue systemic toxicity in our previous study (5-7). Animals were fed caffeine dissolved in water via intra-gastric gavage once daily in the morning (between 9 a.m. and 11 a.m.), and animals in the control group (CT) received the same volume of distilled water at the same time. The developmental stages of the rats were selected on the basis of previously published studies to ensure that any effects of caffeine on skeletal development could be assessed by age. The immature group includes both the juvenile (days 21 35) and peripubertal (days 35 55 ) periods (12). The eighth week of post-natal life (approximately 63 days) marks the transition period from peripuberty to young adult, and adulthood begins at approximately 7 - 8 months of age (approximately 210 days), when skeletal growth slows down in male rats (15). Animals were examined for clinical signs and weighed daily. Food intake was monitored weekly, and the daily intake was calculated by dividing the amount of food consumed in a week by seven. Body weight was measured to the nearest $0.1 \mathrm{~g}$ with an electronic scale (Dretec Corp., Seoul, Korea), and recorded on the first day before caffeine feeding (initial) until the final day of the experiment. All animals were euthanized $24 \mathrm{hrs}$ after the last dose of caffeine, following established protocols and ethical procedures. Animals were anesthetized by isoflurane inhalation (Forane solution, Choongwae Pharma Corp., Seoul, Korea), and killed by cervical dislocation under deep anesthesia.

Dual-energy X-ray absorptiometry (DXA). All animals were evaluated for body composition at the end of the experiment with DXA scanner (Discovery W QDR series, Hologic Inc., Bedford, MA, USA) using software specifically for small animal analysis. Application of this software has been validated in very young animals and is an excellent noninvasive technique for measuring fat and lean tissue in the rat. Bone mineral content (BMC, g), bone mineral density (BMD, $\mathrm{g} / \mathrm{cm}^{2}$ ), lean body mass and body fat were analyzed. Vertebral column length $(\mathrm{cm})$ was measured from the upper border of the $1^{\text {st }}$ cervical vertebra to the lower border of the $4^{\text {th }}$ sacral vertebra. Animals were anesthetized by isoflurane inhalation during examination.

Organ preparation. At the end of the experiment, the femurs and tibiae were dissected from each rat, cleaned of fat, muscle and connective tissue, then weighed and measured from the top of the plateau to the bottom of the lateral malleolar process with a precision digital caliper $( \pm 0.03 \mathrm{~mm}$; NA500-150S, Bluebird, Zhejiang, China), as described by Weinreb et al (16). In addition, the reproductive organs (testes, prostate, seminal vesicle and epididymis), and the adrenal glands were dissected and weighed with an electronic scale to the nearest $0.001 \mathrm{~g}$ (Adventure ${ }^{\mathrm{TM}}$ electronic balances, AR1530, OHAUS Corp., NJ, USA) and their morphology was grossly evaluated.

Statistical analyses. Data for each group are expressed as mean \pm standard deviation (SD). All data were analyzed using SPSS ver. 10.0 for Windows (SPSS Inc., Chicago, IL, USA). Statistical significance was determined by KruskalWallis one-way analysis of variance or two-way ANOVA (analysis of variance) for multiple group comparisons, and the Mann-Whitney $U$ test for two-group comparisons. Significance was accepted at $p<0.05$.

\section{RESULTS}

Body weight and food consumption. The initial weight of the immature group was $50 \pm 2 \mathrm{~g}$ and the initial weight of the young adult group was $323 \pm 2$ g. Body weight was increased with age for both groups throughout the experimental period. Caffeine caused a significant decrease in body weight gain after 1 week in both the immature groups 
(A)

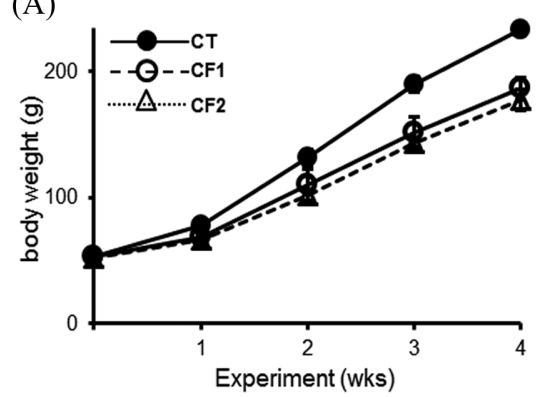

(C)

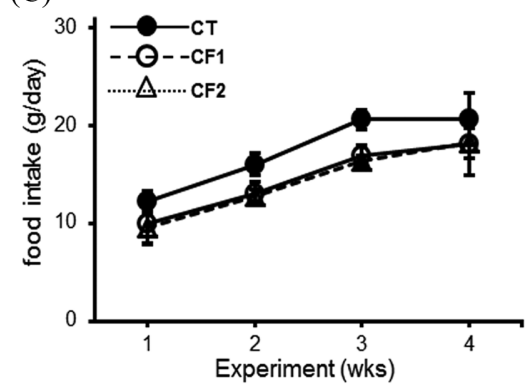

(B)

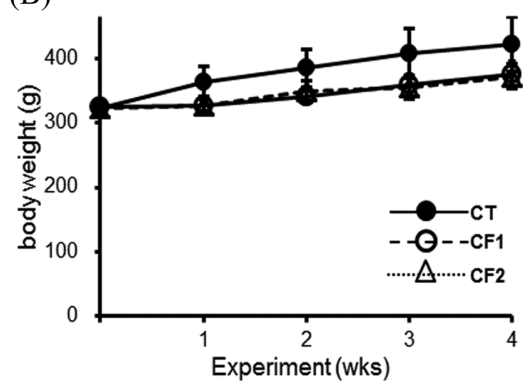

(D)

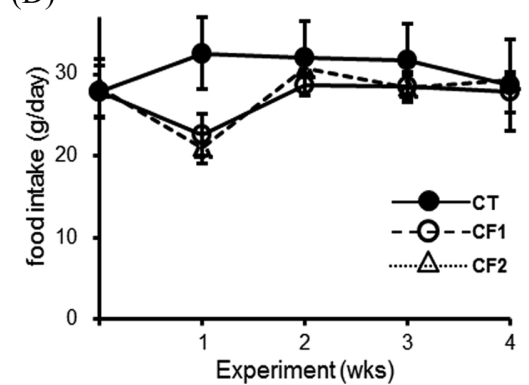

Fig. 1. The effects of exposure to caffeine on body weights and food intake in immature and young adult rats. Average body weights in (A) the immature group ( $p<0.0001$ vs. CT after 1 week) and (B) the young adult group for each week of the study period ( $p<0.01$ vs. CT after 1 week). Average daily food intake in (C) the immature group ( $p<0.05$ vs. CT) and (D) the young adult group was calculated by dividing the amount of food consumed over one week by seven, and is depicted here for each week. Values are mean \pm SD ( $\mathrm{N}=5 / \mathrm{group}$ ). Filled circle, CT (control); open circle, CF1 (caffeine $120 \mathrm{mg} / \mathrm{kg}$ per day); open triangle, CF2 (caffeine $180 \mathrm{mg} / \mathrm{kg}$ per day). Two-way ANOVA test was used for statistical analyses.

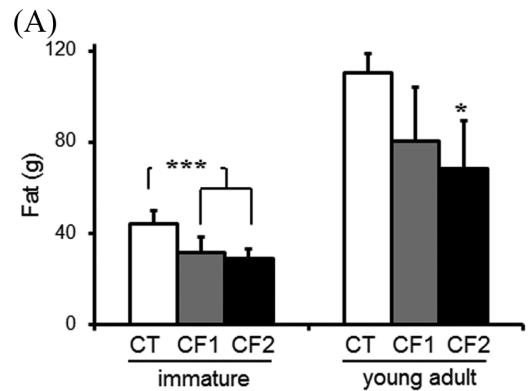

(C)

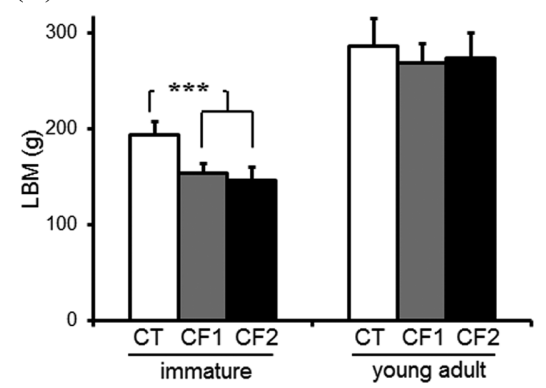

(B)

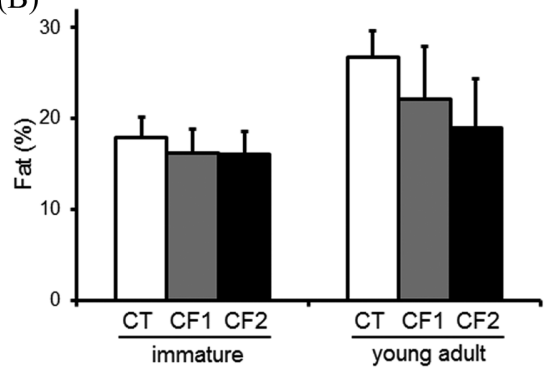

(D)

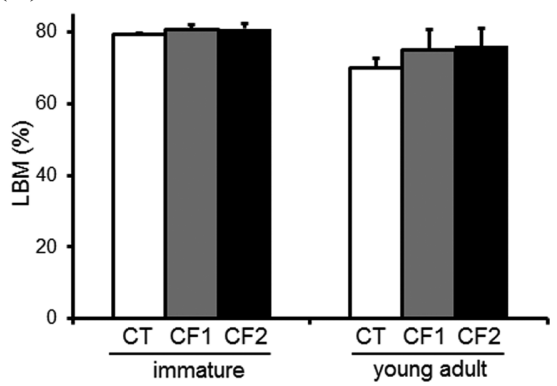

Fig. 2. The effects of exposure to caffeine on total body fats and lean body masses in immature and young adult rats, as determined by DXA. Comparison of (A) total body fat (g), (B) fat \%, (C) lean body mass (LBM, g) and (D) LBM \% in control and caffeine-fed, immature and young adult rats after 4 weeks. Fat \%, total body fat divided by total body mass; LBM \%, LBM divided by total body mass. Values are mean $\pm S D\left(N=5 /\right.$ group). $C T$, control; $C F 1$, caffeine $120 \mathrm{mg} / \mathrm{kg}$ per day; $C F 2$, caffeine $180 \mathrm{mg} / \mathrm{kg}$ per day. ${ }^{*} p<0.05$, ${ }^{* * *} p<0.001$ vs. CT. Statistical analyses were performed by Kruskal-Wallis one-way analysis of variance for the multiple group comparisons, and the Mann-Whitney $U$ test for two-group comparisons. 
$(p<0.0001$ vs. CT) and the young adult groups $(p<0.01$ vs. CT; Fig. 1A, 1B). A profound decrease in food consumption was noted in caffeine-fed animals in the immature group, and this persisted until the end of the experiment ( $p<0.05$ vs. CT; Fig. 1C). In the young adult group, however, a profound decrease in food intake was noted only at 1 week, and thereafter no difference was observed between the caffeine-fed young adult groups and control animals (Fig. 1D). The dose of caffeine (120 or $180 \mathrm{mg} / \mathrm{kg}$ per day) had no effect on either body weight or food intake in either immature or young adult rats.

Body composition. In immature animals, total body fat (Fig. 2A) was significantly decreased in caffeine-fed groups $(p<0.001$ vs. CT); however, the percentage of body fat was
Table 1. Bone mineral content and density, determined by DXA

\begin{tabular}{llcl}
\hline \hline & Group & BMC $(\mathrm{g})$ & BMD $\left(\mathrm{g} / \mathrm{cm}^{2}\right)$ \\
\hline \multirow{4}{*}{ Immature } & CT & $7.31 \pm 0.51$ & $0.144 \pm 0.01$ \\
& CF1 & $5.72 \pm 0.47^{* * *}$ & $0.127 \pm 0.01^{\text {*** }}$ \\
& CF2 & $5.44 \pm 0.30^{* * *}$ & $0.121 \pm 0.01^{* * *}$ \\
\hline \multirow{3}{*}{ Young adult } & CT & $13.30 \pm 1.33$ & $0.177 \pm 0.01$ \\
& CF1 & $11.18 \pm 0.64^{* * *}$ & $0.174 \pm 0.00$ \\
& CF2 & $10.84 \pm 0.67^{* * *}$ & $0.175 \pm 0.01$ \\
\hline
\end{tabular}

Values are mean $\pm S D(N=5$ /group). $B M C$, bone mineral content; $\mathrm{BMD}$, bone mineral density. CT, control; CF1, caffeine $120 \mathrm{mg} / \mathrm{kg}$ per day; CF2, caffeine $180 \mathrm{mg} / \mathrm{kg}$ per day. ${ }^{* *} p<0.01$ vs. $C{ }^{* * * *} p<$ 0.001 vs. CT. Statistical analyses were performed by Kruskal-Wallis one-way analysis of variance for multiple group comparisons, and the Mann-Whitney $U$ test for two-group comparisons.
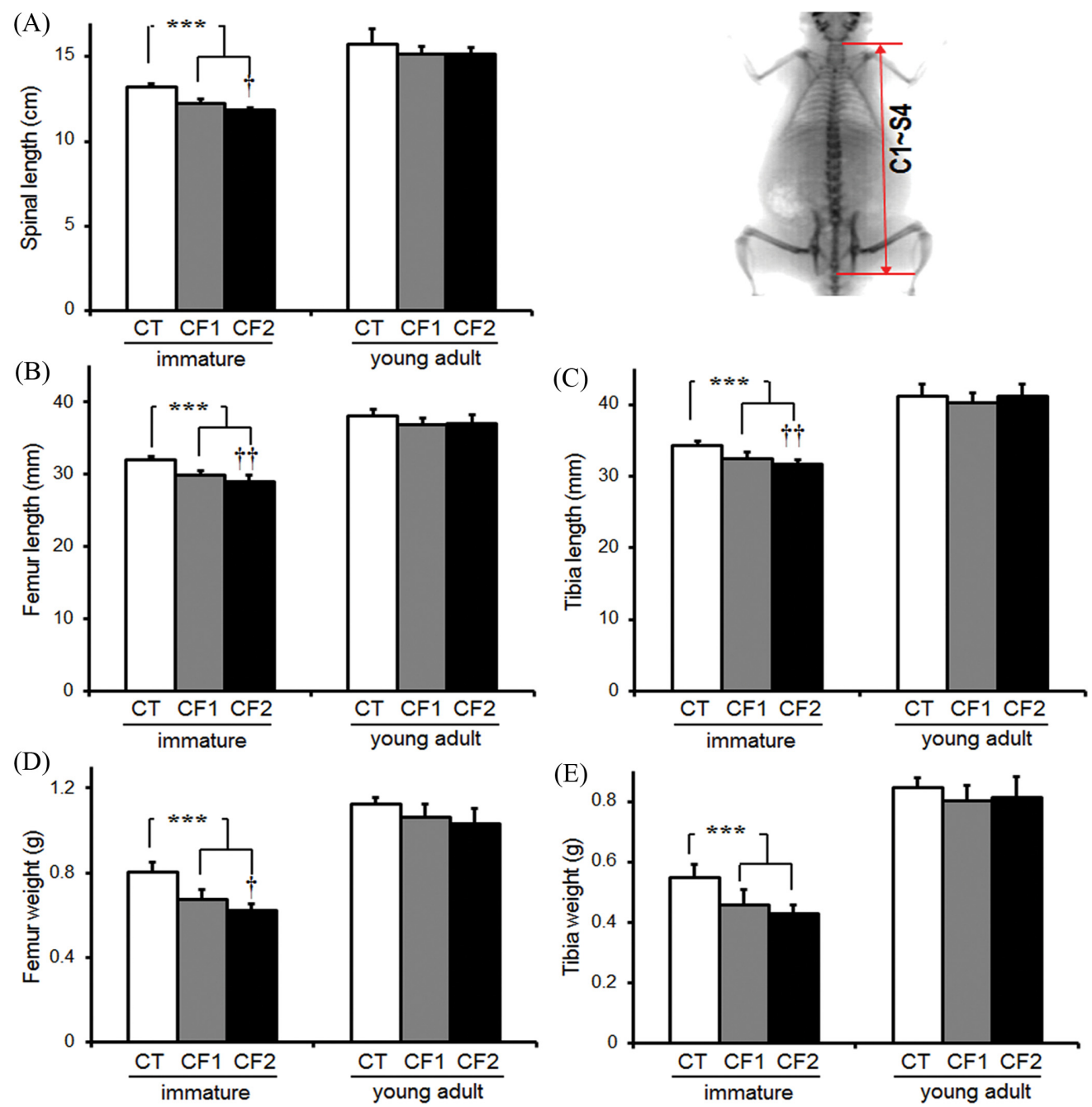

Fig. 3. The effects of exposure to caffeine on the long bones of immature and young adult rats. (A) The length of the vertebral column was measured from the DXA image, as indicated in the right-hand panel. $(B, C)$ Mean length $(\mathrm{mm})$ and weight $(\mathrm{g})$ of $(\mathrm{D})$ femurs and (E) tibiae. Values are mean \pm SD. CT, control; CF1, caffeine $120 \mathrm{mg} / \mathrm{kg}$ per day; CF2, caffeine $180 \mathrm{mg} / \mathrm{kg}$ per day. ${ }^{* * *} p<0.001 \mathrm{vs} . \mathrm{CT}$; ${ }^{\dagger} p<0.05,{ }^{\dagger \dagger} p<0.01, \mathrm{CF} 1$ vs. CF2. Statistical analyses were performed by Kruskal-Wallis one-way analysis of variance for multiple group comparisons, and the Mann-Whitney $U$ test for two-group comparisons. 
not significantly different between the control and caffeinefed groups (Fig. 2B). Likewise, a significant decrease in LBM was found in caffeine-fed rats in the immature group $(p<0.001$ vs. CT; Fig. 2 C), but there was no significant difference in the percentage of LBM (Fig. 2D). In the young adult group we observed relative decreases in total body fat and body fat percentage in caffeine-fed animals (Fig. 2A), and there was no difference in LBM or LBM percentage between control and caffeine-fed groups (Fig. 2C, 2D). These results indicate that exposure to caffeine causes a proportional reduction in body fat and LBM in immature animals, whereas in young adult animals, caffeine exposure causes the loss of body fat but not LBM.

Whole-body BMC and BMD is summarized in Table 1. In immature rats, exposure to caffeine significantly decreased both BMC ( $p<0.001$ vs. CT) and BMD $(p<0.001$ vs. CT). In caffeine-fed young adult rats, BMC was also significantly reduced ( $p<0.01$ vs. CT), but BMD was no different to the control group.

Length and weight of long bones. The length of the vertebral column was measured as an indicator of axial growth, because vertical growth of body is known to follow the same process as that of the long bones (17). In immature animals exposed to caffeine, the length of the long bones was significantly shorter compared to controls (Fig. 3A-3C; Supplemental Fig. 1A), including the spine $(p<0.001$ vs. CT; $p<0.05, \mathrm{CF} 1$ vs. CF2), femur and tibia $(p<0.001$ vs. CT; $p<0.01, \mathrm{CF} 1$ vs. CF2). In addition, the weight of the femur and tibia was significantly decreased in immature rats exposed to caffeine in comparison to control rats (Fig. 3D, 3E). Young adult animals, however, showed no differences in the length or weight of the long bones between the control and caffeine-fed groups (Supplemental Fig. 1B).

Weight and size of reproductive organs. The testes were removed and weighed at the end of the experiment to investigate the effect of caffeine on the reproductive organs. The testes from immature animals exposed to caffeine were significantly smaller and lighter in comparison to the testes from control animals, as shown clearly in Fig. 4A and the upper part of Fig. 4B. However, exposure to caffeine had no effect on the size or weight of testes in young adult animals (Fig. 4B, lower). The sex accessory organs, including the prostate, seminal vesicles and epididymis, were also weighed and the data are summarized in Table 2 (with representative images shown in Supplemental Fig. 2). Overall, exposure to caffeine decreased the weight of all sex accessory organs in both immature and young adult animals, although signifi-

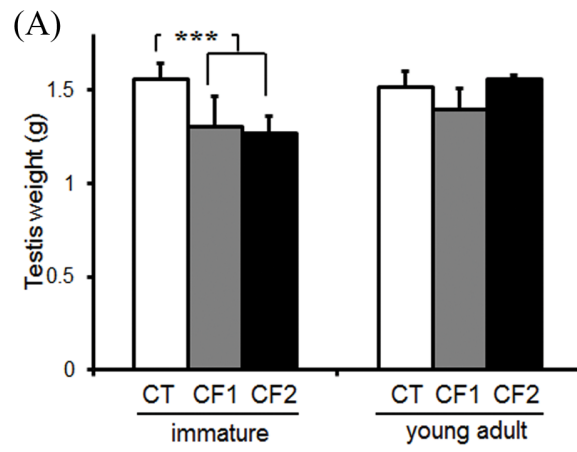

(B)
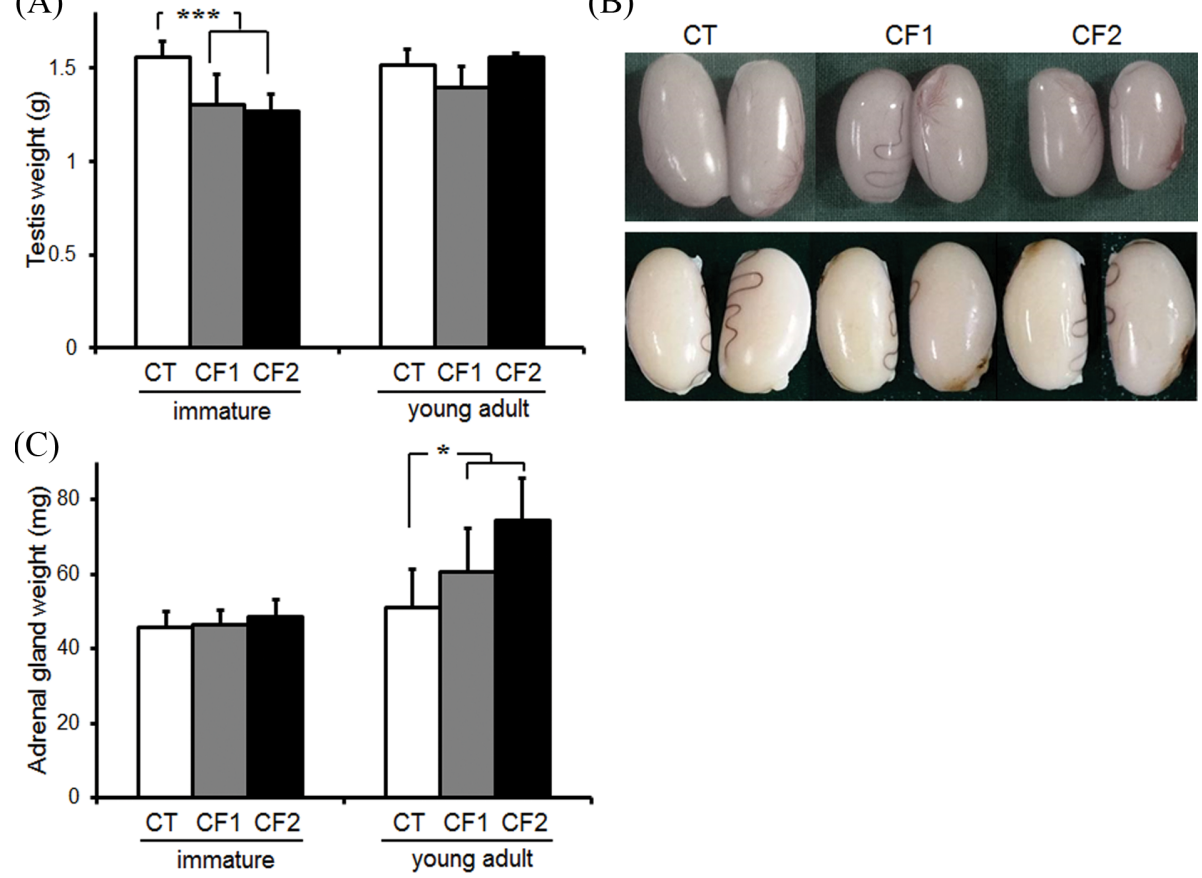

Fig. 4. The effects of exposure to caffeine on the weights of the testes and the adrenal glands in immature and young adult rats. (A) The mean absolute weight of the testes. ${ }^{* * *} p<0.001$ vs. CT. (B) Representative images of testes dissected from the control (upper panel) and caffeine-fed (lower panel) animals. From the left, testes from each group are shown: control (CT), caffeine $120 \mathrm{mg} / \mathrm{kg}$ per day (CF1), and caffeine $180 \mathrm{mg} / \mathrm{kg}$ per day (CF2). (C) The mean absolute weight of the adrenal glands. Values are mean \pm SD. ${ }^{* * *} p<0.001,{ }^{*} p<0.05$ vs. CT. Statistical analyses were performed by Kruskal-Wallis one-way analysis of variance for multiple group comparisons, and the Mann-Whitney $U$ test for two-group comparisons. 
Table 2. The dose-dependent effect of caffeine on the weights of reproductive organs in immature and young adult rats

\begin{tabular}{lllll}
\hline \hline \multirow{2}{*}{ Group } & & Weight $(\mathrm{mg})$ & \\
\cline { 3 - 4 } & & Prostate & SV & Epi \\
\hline \multirow{3}{*}{ Immature } & CT & $338.5 \pm 94.04$ & $234.0 \pm 35.08$ & $228.5 \pm 8.58$ \\
& CF1 & $254.5 \pm 13.43$ & $156.3 \pm 48.75$ & $192.3 \pm 14.24$ \\
& CF2 & $178.0 \pm 31.11$ & $135.3 \pm 24.25$ & $174.5 \pm 7.93$ \\
\hline \multirow{2}{*}{ Young adult } & CT & $637.4 \pm 89.48$ & $503.3 \pm 112.32$ & $562.6 \pm 42.76$ \\
& CF1 & $488.8 \pm 31.85^{*}$ & $409.4 \pm 94.66$ & $510.7 \pm 14.50^{*}$ \\
& CF2 & $555.3 \pm 99.82$ & $428.3 \pm 81.44$ & $510.5 \pm 28.66$ \\
\hline
\end{tabular}

Values are expressed as mean $\pm S D(N=5 / g r o u p)$. SV, seminal vesicle; Epi, epididymis. CT, control; CF1, caffeine $120 \mathrm{mg} / \mathrm{kg}$ per day; CF2, caffeine $180 \mathrm{mg} / \mathrm{kg}$ per day. ${ }^{*} p<0.05$ vs. CT. Statistical analyses were performed by Kruskal-Wallis one-way analysis of variance for multiple group comparisons, and the Mann-Whitney $U$ test for two-group comparisons.

cance was not obtained due to high individual variation.

Weight of the adrenal glands. Caffeine stimulates sympathetic responses of the nervous system by increasing the secretion of glucocorticoid and catecholamine from the adrenal gland under conditions of chronic stress (18). Therefore, we investigated whether caffeine causes any changes to the weight of the adrenal glands. As shown in Fig. 4C, the weight of the adrenal glands was similar in immature animals regardless of caffeine intake, whereas exposure to caffeine causes dose-dependent increase in the weight of the adrenal glands in young adult animals ( $p<0.05$, vs. CT).

\section{DISCUSSION}

In the present study, we clearly show that the negative effects of caffeine on the long bones and testes of rats differ according to age at the time of exposure. Although rats are more susceptible to the effects of caffeine during puberty, high doses of caffeine can still affect body composition, bone composition, and the adrenal glands if exposure to caffeine occurs during young adulthood. To our knowledge, the current study is the first to analyze the effects of caffeine on long bones and testes at different developmental stages.

It has been reported that metabolic patterns of caffeine in rats were more closely related to the human compared to the mice and attained blood levels were comparable in both rats and humans (19). In addition, body surface area-based dose calculations are the most appropriate method for extrapolating from animals to humans and they are far superior to simple conversions based on body weight $(20,21)$. Therefore, using a dose calculation based on body surface area, we estimate that the dosages used in this study of rats (120 and $180 \mathrm{mg} / \mathrm{kg}$ per day) are equivalent to approximately 19.4 and $29.1 \mathrm{mg} / \mathrm{kg}$ per day in humans. Considering that safe caffeine intake for humans is less than $100 \mathrm{mg} /$ day for adolescents and $400 \mathrm{mg}$ /day for healthy adults (18), one or two energy drinks can easily lead to a daily caffeine intake above the recommended safe levels.
As body weight is an approximate indicator for general growth in rats (14), we analyzed body weight and food intake. Consistent with previous reports $(5,8)$, we observed significant reductions in both body weight gain and food intake in caffeine-fed immature animals (Fig. 1A, 1C), indicating that reduced body weight gain can be attributed mainly to decreased food intake.

We observed a similar reduction in body weight gain in caffeine-fed young adult animals, but there was not much difference in food intake, except during the first week of exposure (Fig. 1D). Food intake in young adult animals was transiently reduced at 1 week of exposure and then returned rapidly to control levels. This is a contrary to a previous study of adult animals, which reported a sustained reduction in food intake during exposure to caffeine (3); therefore, young adults might respond differently to caffeine exposure than adults. Reduced body weight gain by caffeine exposure seemed to be related to a continuous negative impact on food intake in immature animals (Fig. 1A, 1C). However, in the young adult groups, reduced body weight gain by caffeine exposure might be related to altered metabolic activity, because food intake was similar between the control and caffeine groups during the rest of experimental period. In fact, caffeine is known to increase both metabolic rate and fat oxidation (22).

Significant age-related changes in body composition, including fat and LBM, affect caffeine metabolism (10). In addition to a reduction in body weight gain in caffeine-fed rats, body fat mass was also reduced compared to the control groups, particularly in young adult animals, where it was accompanied by a reduced proportion of fat (Fig. 2). Because fat tissues might act as endocrine tissues in the regulation of reproductive functions by secreting various products (23), reduced body fat might affect growth in children and adolescents, and alter reproductive functions in young adults. In fact, previous studies have shown that exposure to caffeine delays the onset of puberty in immature animals $(6,9)$ and inhibits serum testosterone levels in young adult animals (8). Overall, caffeine has adverse effects on body growth and body fat, rather than muscle mass, during puberty 
and young adulthood, respectively.

Prenatal and prepubertal exposure to caffeine results in retarded growth of the long bones $(5,6,14)$, possibly by inhibition of chondrocyte differentiation in the growth plate (7). The growth of long bones slows between 7 and 8 months of age in the rat (15). Although the young adult animals observed in this study might still be in a growing stage, there was no difference in the length or weight of the long bones between caffeine-fed rats and the control animals (Fig. 3). It is well known that chronic caffeine consumption causes a decrease in bone minerals by increasing calcium loss (4). We observed significant reductions in BMC following exposure to caffeine in both immature and young adult rats, but BMD was significantly reduced only in immature animals (Table 1). The negative effects of caffeine on bones seem to be dependent on the growing rate; that is, when the growth rate is faster the side effects are more severe. However, high caffeine consumption during young adulthood might also result in serious loss of bone minerals, which might increase the risk of osteoporosis and fractures at a young age.

It has been reported that prenatal exposure to caffeine significantly restricts the growth of testes, in part by interrupting the differentiation of the seminiferous cords (8). Likewise, prepubertal exposure to caffeine reduces both the weight of the testes and the size of the seminiferous tubules in immature animals $(9,24)$. Consistent with these previous reports, we observed that caffeine causes a significant decrease in testicular weight in a dose-dependent manner in immature rats (Fig. 4). Because the testes increase in size until 100 125 days of age in the rat (25), it can be assumed that exposure to caffeine inhibits the growth of testes in young adult animals. Contrary to these expectations, however, we did not observe a reduction in the weight of the testes in young adult animals exposed to caffeine. Whether caffeine exposure causes functional or histological changes to the testes therefore warrants further investigations, because testicular atrophy caused by caffeine intake has been recorded in adult animals (8). Caffeine consumption alters circulating androgen levels in humans and animals $(8,9)$, and, therefore, androgen-dependent sex accessory organs might be affected by caffeine exposure. As expected, we observed in the present study that the weights of the prostate, seminal vesicles and epididymis were reduced in the caffeine-fed animals regardless of age, although statistical significance was not obtained due to high individual variation (Table 2). It can therefore be assumed that secretions from the testes might be altered because of high caffeine consumption during puberty as well as during young adulthood.

The adrenal glands are endocrine glands that produce a variety of hormones, including glucocorticoids, which regulate blood glucose levels and osteoblasts. Prenatal exposure to caffeine inhibits the functional development of adrenal glands (26). In the present study, therefore, although the weights of the adrenal glands were comparable in the immature control and caffeine-fed animals (Fig. 4C), caffeine might still have an effect on the function of the adrenal glands because the hypothalamo-pituitary-adrenal axis is still maturing during puberty. Experimental studies of adult animals reported that exposure to caffeine increased the weight and caused histological distortion of the adrenal glands (18). Consistent with this, we also noted a significant increase in the weight of the adrenal glands following exposure to caffeine in young adult animals (Fig. 4C). In addition, caffeine stimulates glucocorticoid production in both in vivo and in vitro studies (26). Given that glucocorticoids reduce osteoblast activity and calcium absorption in the gastrointestinal tract (27), it can be assumed that increased glucocorticoid levels might be the cause of decreased bone minerals.

The present study shows that the negative effects of caffeine on the long bones and testes in rats vary depending on the developmental stages of rats at the time of exposure. Although the long bones and testes are more susceptible to caffeine during puberty, caffeine has negative effects on body fat, bone minerals and the adrenal glands when exposure occurs during young adulthood. There is a need, therefore, to educate the public about the potential dangers of caffeine consumption during puberty and young adulthood.

\section{CONFLICT OF INTEREST}

All authors state that they have no conflicts of interest with any financial organization regarding the material discussed in the manuscript.

\section{ACKNOWLEDGMENTS}

YK and HC participated in the experiments, data collection and analysis; JR, contributed to the design of the study, data analysis, supervision and development of the manuscript. JR takes responsibility for the integrity of the data analysis. All authors read and approved the final manuscript. This work was supported by the Basic Science Research Program through the National Research Foundation of Korea (NRF) funded by the Ministry of Education and Science [grant number NRF-2014R1A1A2053601].

\section{REFERENCES}

1. Seifert, S.M., Schaechter, J.L., Hershorin, E.R. and Lipshultz, S.E. (2011) Health effects of energy drinks on children, adolescents, and young adults. Pediatrics, 127, 511-528.

2. Ishak, W.W., Ugochukwu, C., Bagot, K., Khalili, D. and Zaky, C. (2012) Energy drinks: psychological effects and impact on well-being and quality of life-a literature review. Innov. Clin. Neurosci., 9, 25-34.

3. Yeh, J.K. and Aloia, J.F. (1986) Differential effect of caffeine administration on calcium and vitamin D metabolism in young and adult rats. J. Bone Miner. Res., 1, 251-258. 
4. You, D.C., Kim, Y.S., Ha, A.W., Lee, Y.N., Kim, S.M., Kim, C.H., Lee, S.H., Choi, D. and Lee, J.M. (2011) Possible health effects of caffeinated coffee consumption on Alzheimer's disease and cardiovascular disease. Toxicol. Res., 27, 7-10.

5. Shin, J., Choi, Y., Kim, J., Yu, A.R., Shin, J.S., Choi, Y.Y. and Roh, J. (2015) High doses of caffeine reduce in vivo osteogenic activity in prepubertal rats. J. Anat., 227, 10-20.

6. Choi, Y.Y., Choi, Y., Kim, J., Choi, H., Shin, J. and Roh, J. (2016) Peripubertal caffeine exposure impairs longitudinal bone growth in immature male rats in a dose- and time-dependent manner. J. Med. Food, 19, 73-84.

7. Choi, H., Choi, Y., Kim, J., Bae, J. and Roh, J. (2017) Longitudinal bone growth is impaired by direct involvement of caffeine with chondrocyte differentiation in the growth plate. $J$. Anat., 230, 117-127.

8. Dorostghoal, M., Erfani Majd, N. and Nooraei, P. (2012) Maternal caffeine consumption has irreversible effects on reproductive parameters and fertility in male offspring rats. Clin. Exp. Reprod. Med., 39, 144-152.

9. Park, M., Choi, Y., Choi, H., Yim, J.Y. and Roh, J. (2015) High doses of caffeine during the peripubertal period in the rat impair the growth and function of the testis. Int. J. Endocrinol., 2015, 368475.

10. Kalyani, R.R., Corriere, M. and Ferrucci, L. (2014) Agerelated and disease-related muscle loss: the effect of diabetes, obesity, and other diseases. Lancet Diabetes Endocrinol., 2, 819-829.

11. Gluckman, P.D. and Hanson, M.A. (2006) Evolution, development and timing of puberty. Trends Endocrinol. Metab., 17, 712.

12. Marty, M.S., Chapin, R.E., Parks, L.G. and Thorsrud, B.A. (2003) Development and maturation of the male reproductive system. Birth Defects Res. B Dev. Reprod. Toxicol., 68, 125136.

13. Olchowik, G., Chadaj-Polberg, E., Tomaszewski, M., Polberg, M. and Tomaszewska, M. (2011) The influence of caffeine on the biomechanical properties of bone tissue during pregnancy in a population of rats. Folia Histochem. Cytobiol., 49, 504511.

14. Tan, Y., Liu, J., Deng, Y., Cao, H., Xu, D., Cu, F., Lei, Y., Magdalou, J., Wu, M., Chen, L. and Wang, H. (2012) Caffeine-induced fetal rat over-exposure to maternal glucocorticoid and histone methylation of liver IGF-1 might cause skeletal growth retardation. Toxicol. Lett., 214, 279-287.
15. Sengupta, P. (2013) The laboratory rat: relating its age with human's. Int. J. Prev. Med., 4, 624-630.

16. Sze, G., Brant-Zawadzki, M., Haughton, V.M., Maravilla, K.R., McNamara, M.T., Kumar, A.J., Aisen, A.M., Dreisbach, J.N., Bradley, W.G., Jr. and Weinreb, J.C. (1991) Multicenter study of gadodiamide injection as a contrast agent in MR imaging of the brain and spine. Radiology, 181, 693-699.

17. Doskocil, M., Valouch, P. and Pazderka, V. (1993) On vertebral body growth. Funct. Dev. Morphol., 3, 149-155.

18. Anderson, N.L. and Hughes, R.N. (2008) Increased emotional reactivity in rats following exposure to caffeine during adolescence. Neurotoxicol. Teratol., 30, 195-201.

19. Babu, K.M., Church, R.J. and Lewander, W. (2008) Energy drinks: the new eye-opener for adolescents. Clin. Pediatr. Emerg. Med., 9, 35-42.

20. Brent, R.L., Christian, M.S. and Diener, R.M. (2011) Evaluation of the reproductive and developmental risks of caffeine. Birth Defects Res. B Dev. Reprod. Toxicol., 92, 152-187.

21. Reagan-Shaw, S., Nihal, M. and Ahmad, N. (2008) Dose translation from animal to human studies revisited. FASEB J., 22, 659-661.

22. Vaughan, R.A., White, A.C., Beam, J.R., Gannon, N.P., Garcia-Smith, R., Salgado, R.M., Bisoffi, M., Trujillo, K.A., Conn, C.A. and Mermier, C.M. (2015) Effect of novel dietary supplement on metabolism in vitro and in vivo. J. Tradit. Complement. Med., 7, 1-8.

23. Inoue, H., Kobayashi-Hattori, K., Horiuchi, Y., Oishi, Y., Arai, S. and Takita, T. (2006) Regulation of the body fat percentage in developmental-stage rats by methylxanthine derivatives in a high-fat diet. Biosci. Biotechnol. Biochem., 70, 1134-1139.

24. Bae, J., Choi, H., Choi, Y. and Roh, J. (2017) Dose- and timerelated effects of caffeine on the testis in immature male rats. Exp. Anim., 66, 29-39.

25. Robb, G.W., Amann, R.P. and Killian, G.J. (1978) Daily sperm production and epididymal sperm reserves of pubertal and adult rats. J. Reprod. Fetil., 54, 103-107.

26. Xu, D., Zhang, B., Liang, G., Ping, J., Kou, H., Li, X., Xiong, J., Hu, D., Chen, L., Magdalou, J. and Wang, H. (2012) Caffeine-induced activated glucocorticoid metabolism in the hippocampus causes hypothalamic-pituitary-adrenal axis inhibition in fetal rats. PLOS ONE, 7, e44497.

27. Christakos, S., Dhawan, P., Porta, A., Mady, L.J. and Seth, T. (2011) Vitamin D and intestinal calcium absorption. Mol. Cell. Endocrinol., 347, 25-29. 